\title{
Estrategias comunicativas y tareas de interacción oral en L3 presenciales y en línea
}

\section{(Communication strategies and face-to-face and online L3 spoken interaction tasks)}

\author{
Lucrecia Keim Cubas \\ Gemma Delgar Farrés \\ Universidad de Vic-Universidad Central de Cataluña, UVic-UCC (España)
}

DOI: http://dx.doi.org/10.5944/ried.22.2.22868

Cómo referenciar este artículo:

Keim, L. C., y Delgar, G. F. (2019). Estrategias comunicativas y tareas de interacción oral en presenciales y en línea. RIED. Revista Iberoamericana de Educación a Distancia, 22(2), pp. 225-244. doi: http://dx.doi.org/10.5944/ried.22.2.22868

\section{Resumen}

Durante la realización de tareas orales de aula, tanto presenciales como en línea, los aprendientes se enfrentan a importantes retos comunicativos que les llevan a actuar de forma estratégica. En el presente artículo se compara el uso de estrategias comunicativas mientras dos parejas de estudiantes de francés y dos de alemán como lenguas adicionales realizan ocho tareas orales de forma presencial y en línea mediante videoconferencia a fin de estudiar la posible incidencia de la modalidad, la tipología de tarea y la lengua sobre el uso de estrategias comunicativas. Las tareas de tipo jigsaw y ranking se grabaron y transcribieron con el programa CLAN y el enfoque metodológico aplicado ha sido cualitativo. En nuestros datos, la tarea de tipo jigsaw suscitó sobre todo el uso de estrategias comunicativas relacionadas con procesos de clarificación, mientras que la tarea de tipo ranking favoreció las estrategias relacionadas con la reelaboración del discurso. Por otro lado, hay ligeras diferencias entre el alumnado de francés y el de alemán en el momento de activar estrategias compensatorias (búsqueda, petición de ayuda, cambio de código). En todos los casos las estrategias se activan de manera articulada tejiendo secuencias interactivas complejas. Así pues, los resultados de nuestro estudio apuntan a que, con excepción de la gestualidad icónica al activar la estrategia mímica, la modalidad no influye de forma destacable sobre el uso de estrategias. Sin embargo, tanto la tipología de tarea como la lengua de aprendizaje parecen haber tenido efecto sobre las estrategias activadas. 
Palabras clave: educación a distancia; estrategias comunicativas; lengua francesa; lengua alemana; comunicación sincrónica.

\begin{abstract}
When carrying out spoken interaction tasks, whether face-to-face or online, learners face considerable communicative challenges which prod them into responding strategically. This article compares the use of communicative strategies as two pairs of students of French and two pairs of students of German as additional languages carry out eight face-to-face and online spoken interaction tasks, the latter via videoconferencing, in order to examine the possible influence of modality, task typology, and language on communicative strategies used. Jigsaw and ranking tasks were recorded and transcribed using the CLAN programme, while the methodological approach applied was qualitative. Our data show that the jigsaw task elicited mostly the use of communication strategies with a view to clarification, while the ranking task tended toward strategies involving discourse reformulation. Furthermore, there appeared slight differences between students of French and students of German when resorting to compensatory strategies (searching, asking for help, code-switching). In all cases strategies were employed articulately forming complex communicative sequences. Thus, the results of our study indicate - with the exception of iconic gesturing utilised in mime strategy - modality does not significantly impact on the use of strategies. Nevertheless, both task typology and the particular language being learned may have an effect on strategies utilised.
\end{abstract}

Keywords: distance learning; communication strategies; French; German; synchronous communication

La práctica de la interacción oral en lenguas extranjeras mediada por ordenador ha sido objeto de estudio desde diversas motivaciones y tradiciones investigadoras y con objetivos de diferente tipo. Sin embargo, y tal como afirma Wigham (2017), existen aún pocos estudios sobre el potencial de la práctica de la interacción oral mediante videoconferencia al realizar tareas comunicativas de aula con fines pedagógicos. Nuestro estudio está focalizado en la resolución de tareas de interacción oral de forma presencial y a distancia y, más concretamente, en la activación de estrategias comunicativas durante este tipo de interacción. Así pues, se inscribe en la tradición de investigaciones sobre datos de interacción oral realizadas con un enfoque interaccionista y centradas en la negociación de significado (Yanguas, 2010). Pero en nuestro estudio nos ha interesado tener en cuenta la ecología del entorno comunicativo, es decir, de la modalidad, y los posibles efectos que pudiera tener la multimodalidad sobre la activación de estrategias comunicativas para la resolución de las tareas. Nos situamos así en la línea de los estudios de Develotte, Kern y Lamy (2011), Guichon y Cohen (2016), Hampel y Stickler (2012), Knight, Barberà y Appel (2017) y Wigham (2017), entre otros. En anteriores estudios piloto (Delgar, 2015; Keim y Tortadès, 2015) ya habíamos constatado este potencial. Sin embargo, se había visto la necesidad de exponer a los mismos sujetos a una mayor 
variedad de tareas. Siguiendo a Bygate (2015, pp. 15-16), entendemos que las tareas son "classroom activities intended to develop language learning, in which learners use language, orally or in writing, with a focus on meaning, in order to create, communicate, or derive non-linguistic understanding - information, feelings, ideas, or social relations". Como es sabido, el formato de la tarea puede influir sobre el tipo de producción que suscita. Según Pica et al. (1993), las tareas de tipo jigsaw e information-gap, con intercambio de información, son las que más propician la negociación de significado y la interacción. Por otra parte, según Baralt, Gilabert y Robinson (2014), la complejidad cognitiva de una tarea está determinada por dos componentes fundamentales, las variables que dirigen la atención hacia los aspectos lingüísticos de la tarea (resource-directing) y aquellas otras que dispersan la atención hacia aspectos no lingüísticos (resource-dispersing). En el momento de escoger el diseño de las tareas y definir el diseño de la recogida de datos, hemos tenido en cuenta los aspectos que se acaban de describir.

Durante la resolución de las tareas, los aprendientes se enfrentan a importantes retos comunicativos que les llevan a actuar de forma estratégica. Entre las numerosas publicaciones sobre estrategias comunicativas durante la realización de tareas orales son pocos los estudios recientes en los que se haya analizado de forma específica la influencia de la tipología de tarea sobre el uso de estrategias comunicativas. Khan (2010) detecta diferencias en el uso de estrategias en tres tipos de tareas (picture story, art description e information-gap) pero tan solo significativas por lo que se refiere a las estrategias que define como compensatorias. Rosas (2018), a su vez, detecta que los retos lingüísticos y el contexto situacional influyen sobre la selección de estrategias durante la realización de las tareas de tipo jigsaw y, en cambio, son los retos cognitivos asociados a la tarea de conversación libre los que influyen sobre la selección de estrategias. Las taxonomías de tareas generadas en los estudios realizados son a su vez numerosas. Para nuestro trabajo hemos optado por recurrir como punto de partida a una taxonomía preexistente y hemos seleccionado la taxonomía validada por Khan (2010), y de forma particular hemos tenido en cuenta su propuesta de estrategias comunicativas interaccionales y compensatorias. Entendemos con Khan que son estrategias interaccionales todas aquellas estrategias comunicativas que se activan una vez detectado un problema de comunicación propio o del interlocutor. Con estrategias compensatorias, se hace referencia a todos aquellos mecanismos activados para intentar compensar déficits de recursos (Dörnyei y Kormos, 1998) como pueden ser problemas de léxico, gramaticales o de articulación. En muchos estudios se analiza el uso de estrategias introduciendo la variable del nivel de los aprendientes. En nuestro estudio, ya que optamos por mantener la variable de nivel estable (todos los estudiantes tenían un nivel B1), este factor no lo hemos contemplado. 


\section{ESTUDIO}

\section{Objetivos y metodología}

El objetivo de esta investigación es el estudio de las interacciones sincrónicas por videoconferencia entre estudiantes de alemán y de francés como lengua extranjera (L3). Concretamente, nos proponemos analizar cuál es la repercusión del dispositivo en la realización de dos tipos de tareas pedagógicas, una de tipo jigsaw y otra de tipo ranking, desde el punto de vista del uso de las estrategias de comunicación por parte de los aprendientes. Así pues, las preguntas que constituyen el punto de partida de nuestra investigación son las siguientes:

1. ¿Existen diferencias entre las estrategias comunicativas que se activan durante las tareas de interacción oral en la modalidad presencial y en línea?

2. ¿Son distintas las estrategias de comunicación usadas en las tareas del tipo jigsaw y en las tareas del tipo ranking?

3. ¿Existen diferencias a nivel de las estrategias utilizadas por los aprendientes de alemán y los de francés?

Por lo que se refiere al corpus de trabajo, consta de dieciséis grabaciones de video y audio. Los participantes en la investigación fueron un total de ocho estudiantes de cuarto curso del grado de Traducción e Interpretación (2014-15), cuatro de lengua alemana y cuatro de lengua francesa, con un nivel B1. Todos ellos se conocían y tenían experiencia previa de uso de herramientas de comunicación a distancia ${ }^{1}$. Dado que en nuestro estudio las tareas no formaban parte de la actividad de aula habitual, los estudiantes participaron de forma voluntaria en la práctica.

Se constituyeron cuatro díadas, dos para el alemán y dos para el francés, y cada díada realizó los dos tipos de tareas a distancia y de manera presencial. Además, para evitar la familiarización con la tarea, se trabajó con dos tareas de tipo jigsaw y dos de tipo ranking similares (véase la tabla 1).

Tabla 1. Diseño de la recogida de datos

\begin{tabular}{|l|c|c|c|}
\hline \multicolumn{2}{|c|}{ Día 1 } & \multicolumn{2}{c|}{ Día 2} \\
\hline & Díada A & & Díada A \\
\hline En línea & Jigsaw 1 & Presencial & Jigsaw 2 \\
\hline Presencial & Ranking 1 & En línea & Ranking 2 \\
\hline & Díada B & & Díada B \\
\hline En línea & Jigsaw 2 & Presencial & Jigsaw 1 \\
\hline Presencial & Ranking2 & En línea & Ranking 1 \\
\hline
\end{tabular}


Como es habitual, en las tareas del tipo jigsaw los estudiantes debían llegar a un acuerdo sobre una serie de objetos a seleccionar (seis entre doce en total). Los objetos escogidos, si bien son habituales en la vida cotidiana, no formaban parte en su mayoría del léxico de B1 trabajado por este alumnado. Por lo que respecta a las del tipo ranking, en un caso, la realización de la tarea consistía en ordenar una lista común de diez acciones de la más a la menos ética, y, en el otro, en organizar, por orden de importancia y de manera argumentada, diez factores claves para encontrar trabajo ${ }^{2}$. Para las tareas por videoconferencia, los participantes recibieron los enunciados justo antes de comenzar por correo electrónico y, para las efectuadas cara a cara, en papel. Por tanto, la tarea no estaba inscrita en una secuenciación determinada de pre- y postarea. Tuvieron cinco minutos para prepararse y la duración de los diálogos se fijó en aproximadamente diez minutos. Las conversaciones se desarrollaron en dos salas de la universidad. Las interacciones en línea se realizaron a través de la herramienta BigBlueButton y las presenciales se filmaron con cámara de vídeo y, además, se grabaron mediante magnetófonos. Las docentes investigadoras se retiraban una vez solventadas las dudas iniciales sobre aspectos técnicos o sobre las indicaciones para realizar la tarea.

Al final de la realización de cada bloque de dos tareas, se efectuó una entrevista retrospectiva (Chamot, 2004) a los estudiantes para saber cuáles habían sido sus percepciones en relación con la realización de las mismas. Durante esta entrevista retrospectiva, se les mostraba la grabación y se les pedía que la hicieran parar cuando quisieran hacer algún comentario.

La metodología aplicada ha sido de carácter cualitativo y se ha tenido en cuenta tanto la perspectiva de las dos investigadoras como la perspectiva émica. Tras unos primeros visionados del conjunto de los datos, optamos por transcribir los siete primeros minutos de cada una de las dieciséis tareas realizadas (112 minutos) con el programa CLAN del proyecto CHILDES (versión 2016) a fin de poder hacer una descripción cuantitativa de determinados fenómenos y posibilitar la comparación entre las interacciones en línea y las interacciones cara a cara (Cosnier y Develotte, 2011). La duración de siete minutos vino marcada por la duración de la conversación más corta. En las transcripciones se introdujo información sobre aspectos paraverbales y no verbales (Mondada, 2016). Con las herramientas del programa CLAN se calcularon el total de palabras, de pausas y de pausas llenas.

A continuación, se procedió a detectar en los datos aquellos segmentos de la interacción que contenían marcas discursivas indicadoras de la activación de estrategias comunicativas por parte de los participantes. Este primer análisis nos llevó a centrarnos en el proceso detallado de categorización tan solo en las estrategias interaccionales y compensatorias (véase anexo). Tal y como se ha expuesto arriba, partíamos de una taxonomía preestablecida de posibles estrategias (Khan, 2010) y de forma particular hemos tenido en cuenta su propuesta de estrategias comunicativas interaccionales y compensatorias, es decir, un total de once estrategias interaccionales y diez compensatorias. A partir del análisis de 
nuestro corpus, añadimos tres estrategias de interacción (interrumpir para ofrecer una alternativa, construcción colaborativa del discurso y petición de espera) y una de tipo compensatorio (simplificación discursiva). Además, optamos por sumar a las compensatorias las estrategias de mímica, que en nuestro análisis se ha de entender exclusivamente como gestualidad icónica, y de evitación de riesgos que figuraban en la clasificación de Khan (2010) como estrategias para mantener el flujo de la interacción. Una vez fijadas las categorías y codificadas todas las estrategias en las transcripciones, nos servimos de la estadística descriptiva para calcular las cifras totales de estrategias y sus medias por tarea y por persona. Con base en estos cálculos, y tras contrastar nuestras observaciones con las entrevistas retrospectivas, se escogieron los segmentos de interacción a analizar de forma cualitativa partiendo de las marcas tanto verbales ("kannst du mir erklären..." o "qu' est ce que c'est ça?") como no verbales (mímica facial o gestualidad icónica) de aquellas estrategias con medias más altas. Los segmentos se analizaron a continuación aplicando el análisis conversacional (González-Lloret, 2015) a fin de detectar posibles patrones de combinación de estrategias y de actuación comunicativa.

\section{Resultados}

A fin de dar respuesta a nuestras preguntas se empezó con la comparación de la cantidad total de estrategias activadas por los cuatro estudiantes de alemán y los cuatro de francés respectivamente durante los siete primeros minutos de realización de las ocho tareas en cada una de las lenguas, dieciséis en total. A nivel global, el comportamiento de ambos grupos de estudiantes es similar si bien el uso normalizado de estrategias por mil palabras muestra un uso ligeramente superior en el caso de los estudiantes de alemán. La media de estrategias por tarea muestra que los dos grupos de estudiantes hacen un uso considerablemente mayor de estrategias compensatorias que de estrategias interaccionales.

Tabla 2. Total de estrategias

\begin{tabular}{|c|c|c|c|c|c|c|}
\hline & $\begin{array}{c}\text { Total de } \\
\text { estrategias } \\
\text { en } 8 \text { tareas } \\
\text { (alemán) }\end{array}$ & \begin{tabular}{|c|} 
Total \\
estrategias/ \\
1000 \\
palabras \\
(alemán)
\end{tabular} & $\begin{array}{l}\text { Media de } \\
\text { estrategias } \\
\text { por tarea } \\
\text { (alemán) }\end{array}$ & $\begin{array}{c}\text { Total de } \\
\text { sestrategias } \\
\text { en } 8 \text { tareas } \\
\text { (francés) }\end{array}$ & $\begin{array}{c}\text { Total } \\
\text { estrategias/ } \\
1000 \\
\text { palabras } \\
\text { (francés) }\end{array}$ & $\begin{array}{l}\text { Media de } \\
\text { estrategias } \\
\text { por tarea } \\
\text { (francés) }\end{array}$ \\
\hline Total & 543 & 120 & 67,9 & 552 & 98,9 & 69 \\
\hline Interaccionales & 171 & 37,8 & 21,4 & 177 & 31,7 & 22,1 \\
\hline Compensatorias & 372 & 82,2 & 46,5 & 375 & 67,2 & 46,9 \\
\hline
\end{tabular}

Nos interesa, sin embargo, contrastar el uso de estrategias de forma diferenciada en función de la tarea y en función de la modalidad en la que se llevó a cabo. Tal y 
como se muestra en las tablas 3 y 4 , vemos como el mayor uso total de estrategias lo provocó la tarea de tipo jigsaw. En el caso del alemán, en la modalidad en línea y en el caso del francés, en la modalidad presencial. Por lo que se refiere a las estrategias interaccionales en esta tipología de tarea las más utilizadas son la clarificación (mediante la paráfrasis o mediante la repetición), la petición de clarificación y la construcción colaborativa del discurso tanto en alemán como en francés. En cuanto a las estrategias compensatorias, cuya frecuencia es notablemente superior, la mímica es la estrategia más usada, sobre todo en la modalidad presencial. En las tareas de tipo ranking, hay algunas diferencias entre las estrategias usadas por el alumnado de alemán y el de francés. En el caso del alemán, vemos como la media de uso de estrategias es más alta en la modalidad en línea que en la presencial. En cambio, el alumnado de francés activa prácticamente la misma cantidad de estrategias en ambas modalidades. En cuanto a la tipología de estrategias, la petición de clarificación y la construcción colaborativa del discurso en el caso del francés y, en el caso del alemán, la construcción colaborativa del discurso y la clarificación mediante la paráfrasis son las estrategias interaccionales más usadas. En cuanto a las compensatorias, lo son las pausas largas y la reestructuración en francés en ambas modalidades. En alemán, en la modalidad en línea las más usadas son la acuñación de palabras, extranjerización y traducción literal, y en la modalidad presencial, la mímica, si bien en ambas modalidades las pausas largas se usan con más frecuencia que en la tarea de tipo jigsaw.

Tabla 3. Medias individuales de estrategias según tarea en alemán y en francés

\begin{tabular}{|c|c|c|c|c|c|c|c|c|c|c|c|c|}
\hline \multirow[t]{2}{*}{$\begin{array}{c}\text { Medias } \\
\text { individuales } \\
\text { de estrategias } \\
\text { según tarea }\end{array}$} & \multicolumn{2}{|c|}{$\begin{array}{c}\text { Jigs onl } \\
\mathbf{N}=4\end{array}$} & \multicolumn{2}{|c|}{$\begin{array}{l}\text { Jigs pres } \\
\quad \mathbf{N}=4\end{array}$} & \multicolumn{2}{|c|}{$\begin{array}{c}\text { Jigs } \\
\text { total } \\
\mathbf{N}=\mathbf{8}\end{array}$} & \multicolumn{2}{|c|}{$\begin{array}{c}\text { Rank onl } \\
\mathbf{N}=4\end{array}$} & \multicolumn{2}{|c|}{$\begin{array}{c}\text { Rank pres } \\
\mathbf{N}=4\end{array}$} & \multicolumn{2}{|c|}{$\begin{array}{l}\text { Rank } \\
\text { Total } \\
\mathbf{N}=\mathbf{8}\end{array}$} \\
\hline & Ale & Fran & Ale & Fran & Ale & Fran & Ale & Fran & Ale & Fran & Ale & Fran \\
\hline Total & 27,5 & 34,75 & 23,75 & 53,5 & 25,63 & 44,1 & 23 & 24,5 & 18,75 & 25,25 & 20,88 & 24,9 \\
\hline Interaccionales & 16,5 & 14,5 & 8,5 & 16,5 & 12,5 & 15,5 & 12,25 & 8,25 & 5,5 & 5 & 8,88 & 6,7 \\
\hline Compensatorias & 11 & 20,25 & 15,25 & 37 & 13,13 & 28,6 & 10,75 & 16,25 & 13,25 & 20,25 & 12 & 18,25 \\
\hline
\end{tabular}

Tabla 4. Medias individuales de estrategias más recurrentes según tarea en alemán y en francés

\begin{tabular}{|l|c|c|c|c|c|c|c|c|}
\hline $\begin{array}{c}\text { Medias individuales de } \\
\text { estrategias más recurrentes } \\
\text { según tarea }\end{array}$ & \multicolumn{2}{c|}{$\begin{array}{c}\text { Jigs onl } \\
\mathbf{N}=\mathbf{4}\end{array}$} & \multicolumn{2}{c|}{$\begin{array}{c}\text { Jigs pres } \\
\mathbf{N}=\mathbf{4}\end{array}$} & \multicolumn{2}{c|}{$\begin{array}{c}\text { Rank onl } \\
\mathbf{N}=\mathbf{4}\end{array}$} & \multicolumn{2}{c|}{$\begin{array}{c}\text { Rank pres } \\
\mathbf{N}=4\end{array}$} \\
\hline Estrategias interaccionales & Ale & Fran & Ale & Fran & Ale & Fran & Ale & Fran \\
\hline Petición de clarificación & 2,25 & 3,25 & 2,5 & 3,25 & 1,5 & 2,5 & 0,25 & 2,75 \\
\hline Clarificación mediante paráfrasis & 4 & 4 & 1,75 & 5,25 & 1,25 & 0,25 & 1,25 & 0 \\
\hline
\end{tabular}




\begin{tabular}{|l|c|c|c|c|c|c|c|c|}
\hline $\begin{array}{c}\text { Medias individuales de } \\
\text { estrategias más recurrentes } \\
\text { según tarea }\end{array}$ & \multicolumn{2}{c|}{$\begin{array}{c}\text { Jigs onl } \\
\mathbf{N}=4\end{array}$} & \multicolumn{2}{c|}{$\begin{array}{c}\text { Jigs pres } \\
\text { N=4 }\end{array}$} & \multicolumn{2}{c|}{$\begin{array}{c}\text { Rank onl } \\
\text { N }=4\end{array}$} & \multicolumn{2}{c|}{$\begin{array}{c}\text { Rank pres } \\
\text { N }=4\end{array}$} \\
\hline $\begin{array}{l}\text { Construcción colaborativa del } \\
\text { discurso }\end{array}$ & 1,25 & 1,5 & 0,75 & 2,75 & 1,5 & 2,5 & 1,25 & 1,5 \\
\hline Clarificación mediante repetición & 2 & 2,25 & 0,5 & 2,25 & 2 & 1,25 & 1,5 & 0,5 \\
\hline Estrategias compensatorias & Ale & Fran & Ale & Fran & Ale & Fran & Ale & Fran \\
\hline Mímica & 3 & 5,75 & 7,5 & 17,25 & 0,5 & 0,5 & 2,25 & 1 \\
\hline Pausas largas & 1,25 & 3 & 0,5 & 3,5 & 1,75 & 7 & 2 & 6 \\
\hline Reestructuración & 0,5 & 2 & 1 & 2,5 & 1 & 2,5 & 1,75 & 3,75 \\
\hline $\begin{array}{l}\text { Acuñación de palabras, } \\
\text { extranjerización, traducción } \\
\text { literal }\end{array}$ & 1,25 & 2,75 & 0,75 & 2 & 3,75 & 1,5 & 0,75 & 2 \\
\hline Búsqueda & 0,5 & 2,5 & 0,5 & 3 & 1 & 1,75 & 0,75 & 2,5 \\
\hline Cambio de código & 1,5 & 1,75 & 0 & 2 & 0,25 & 1,5 & 0,5 & 2,25 \\
\hline Petición de ayuda & 0 & 0 & 1,5 & 1 & 1,5 & 0 & 1,5 & 0 \\
\hline
\end{tabular}

Análisis de la interacción

El análisis más detallado de aquellos segmentos en los que los estudiantes activan las estrategias de uso frecuente comentadas arriba nos muestra que no se activan de forma aislada, sino que se combinan varias estrategias para superar el problema comunicativo y que para ello los estudiantes interactúan entre ellos. Por ejemplo, se aprecia que la petición de clarificación suele ir acompañada de la clarificación mediante la paráfrasis y/o mediante la repetición. Además, la clarificación mediante paráfrasis puede conllevar el uso de la estrategia compensatoria de aproximación y, casi siempre, el uso de la mímica para ilustrar el mensaje verbal.

Así vemos como en el segmento del ejemplo 1 la petición de clarificación lleva a la clarificación con paráfrasis combinada con la estrategia compensatoria mímica que se usa a continuación o de forma paralela a la clarificación. Este uso complementario de estrategias se suele dar en nuestros datos en momentos de negociación de significado originados por una laguna de léxico por parte del interlocutor. 
Ejemplo 1. Jigsaw online díada A

*ANN: $\quad \mathrm{mhm}$ (.) \&=ges:Kopf [asiente] ich habe gedacht dass (.) eine Fischengel @ c ist sehr wirklich@c\&=ges:Gesicht[mira en la pantalla hacia la compañera].

*NAT: $\quad m h m$ ea\& $=$ ges:Gesicht [expresión de incomprensión] können $+/$ kannst $[/ /]$ du $\mathrm{mir}$ erklären über was ist das? \&=ges:Gesicht[mira en la pantalla hacia la compañera] (.).

*ANN: ja (.) \&=ges:Gesicht [mira en la pantalla hacia la compañera] wenn wir Fisch (.) essen wollen (.).

*NAT: ja:

*ANN: wir benutzen diese: (.) Dinge its like@eng its@eng wie [// (.) ein (..)warten Sie bitte (..)

*NAT: ja:

*ANN: $\quad \&=$ lacht wie ein Sto:ck (..)

$\&=$ ges:cor[mimetiza un palo cogido con la mano].

*NAT: $\quad \mathrm{mhm}$.

*ANN: $\quad$ zum Fischen $[<]<\&=1$ acht $x x x\rangle$

"NAT: $\quad<$ ja aber $>[>]=$ ges:Kopf glaubst du dass es ist (.)ea (.) wichtig? (.)

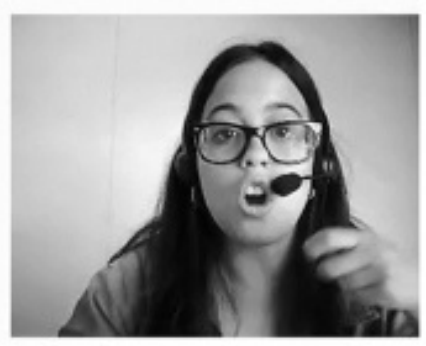

Tras la petición de clarificación por parte de NAT "kannst [//] du mir erklären über was ist das?" que expresa mirando hacia la compañera en pantalla, ANN inicia la clarificación en la cual opta por contextualizar la palabra "Fischangel". Para ello, tras recurrir al diccionario, describe el uso que se le da a una caña de pescar. A pesar de que la compañera va ratificando la recepción (“ja:", "mhm”), finalmente opta por activar una estrategia compensatoria de mímica acompañada de risa. Cuando acaba la compañera confirma la comprensión (“ja”).

En el ejemplo 2, observamos como la petición de clarificación de CAR recibe una respuesta de VIC que pasa primero por la repetición del sustantivo "tondeuse à gazon" para enlazar luego con la paráfrasis que se construye en torno a la aproximación "machine". Estas estrategias se ven reforzadas por el empleo de la mímica que acompaña con los gestos correspondientes las palabras "machine” y "couper", y la onomatopeya "prrrr". 
Ejemplo 2. Jigsaw presencial díada A
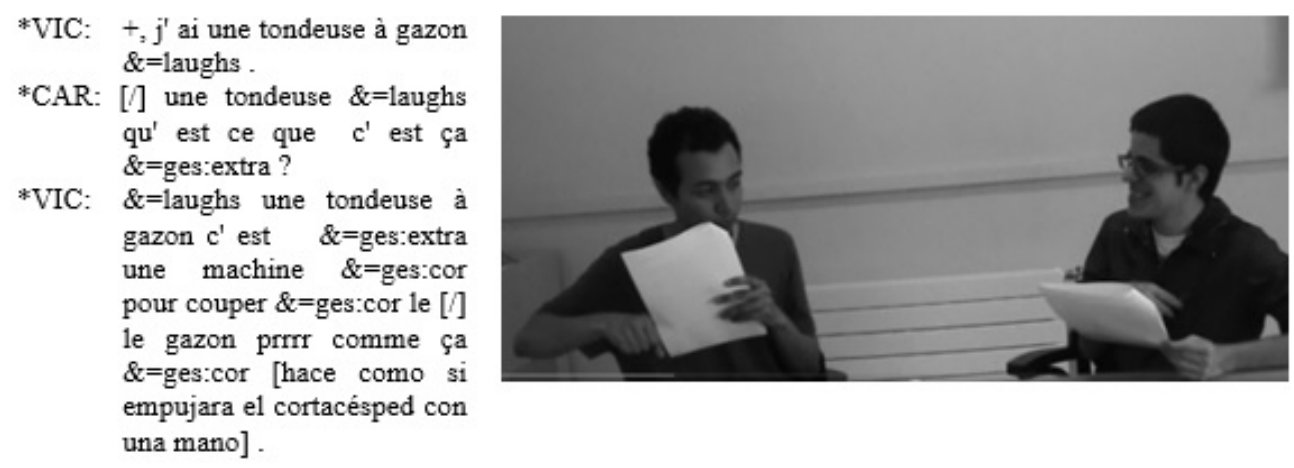

En alguna otra ocasión se combina la petición de ayuda con pausas y mímica:

Ejemplo 3. Ranking presencial díada B

*EST: weil wenn du eine Sekretärin (.) + /.

*CAR: mhm \&=ges: Gesicht [levanta la vista de la hoja y mira directamente a la compañera].

*EST: $\quad+$, bist zum Beispiel und du musst mit (.) \& $=$ ges:cor [abre los brazos y los mueve hacia dentro y afuera como indicando que se comparte el espacio con más personas] $+/$.

${ }^{*} \mathrm{CAR}: \quad++$ mit anderen Leute $[<] \mathrm{xx}$

*EST: $\quad$ mit $[\gg]$

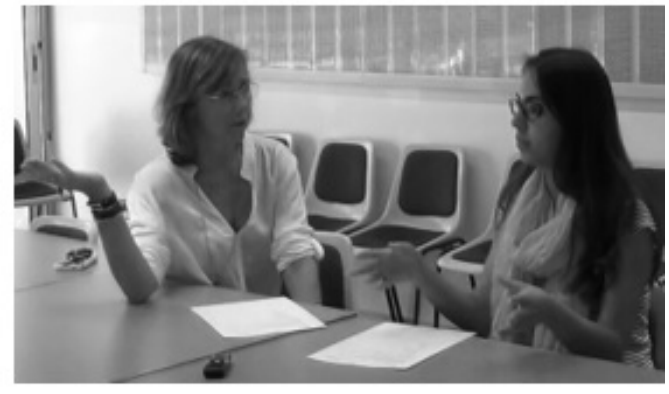

anderen Leute arbeiten (.)

$\&=$ ges:Kopf [vuelve a mirar la hoja].

*CAR: $\quad \mathrm{mhm} \&=$ ges:Kopf [vuelve a mirar la hoja].

*EST: das Aussehen ist wichtig auch (.).

*CAR: $\mathrm{mhm}$.

Es interesante ver como CAR entiende la petición de ayuda que EST realiza mediante gestos icónicos y, una vez que le ofrece una posible solución, EST la retoma lo cual le permite acabar su argumentación. CAR ratifica la recepción de la argumentación de la compañera ("mhm") en dos ocasiones.

Tal y como hemos destacado arriba, en la tarea del tipo ranking presencial la media de uso de la estrategia de reestructuración es más alta que en las otras tareas. El análisis de las transcripciones muestra que se activa sobre todo en momentos de reelaboración del discurso. Esta estrategia compensatoria también se relaciona 
a veces con la construcción colaborativa del discurso como, por ejemplo, en la secuencia del ejemplo 4. Aquí la elaboración compartida del discurso culmina con dos reestructuraciones que reflejan la búsqueda de la estructura lingüística que mejor puede expresar la idea que se desea transmitir.

Ejemplo 4. Ranking presencial díada B

*LAU: oui \&=ges:tête (.) je sais $\&=$ ges:cor $[/]$ je sais ce que tu veux dire mais je crois que $\&=$ ges:extra $+\ldots$

*KAO: oui \& $\&=$ ges:tête .

*KAO: $++[/]$ que $c^{\prime}$ est $\&=$ ges:cor $\&=$ laughs $+\ldots$

*LAU: ++ [] que ce n' est pas \&=ges:tête $[/ / /$ que c' est égal le genre \& $=$ ges:face $\&=$ ges:cor $($.$) bon à mon avis$

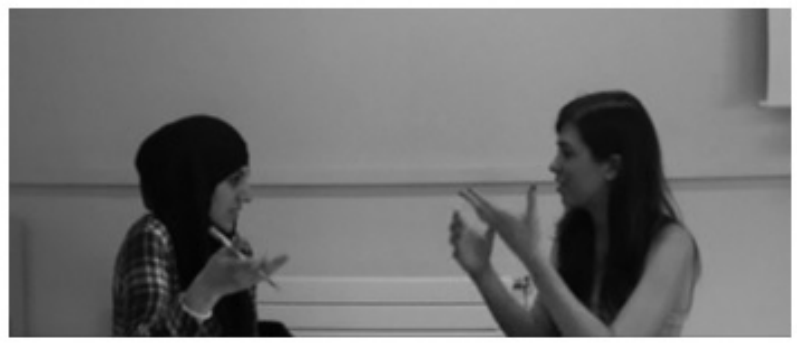
$\&=$ ges:extra .

Cabe decir que en el caso de las tareas realizadas en alemán la reestructuración puede estar motivada por la voluntad de autocorrección gramatical o léxica y se da tanto en la modalidad presencial como en la modalidad en línea.

Ejemplo 5. Ranking online díada A

*ANN: glaubst du es ist sehr important@ cat (.) \&=ges:Kopf [mira a la compañera] important@cat Kontakt [///] wichtige Kontakte haben? (..)

*NAT: ea ich $[/]$ ich $[/]$ ich glaube ea ea (.) mehr oder weniger die gleich $[/ / / /]+/ /$.

*ANN: 0 \&=ges:Kopf.

*NAT: + , das gleichen (.) weil es ist ea $\&=$ ges:Gesicht ea [///] du musst the@eng $[/ / / /]$ die Job (.) ea denken (.)

*ANN: 0 \& =ges:Kopf.

*NAT: welche Job ea willst du arbeit $+/ /$.

*ANN: 0 \& =lacht.

*NAT: + , arbeiten.

*ANN: \&=lacht ja:.
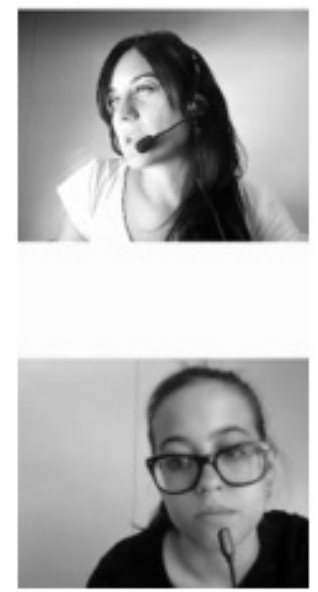
En el ejemplo 5, NAT necesita tres turnos para completar su argumentación que reelabora mientras intercala pausas, pausas llenas y algún cambio de código involuntario que autocorrige. ANN asiente con movimientos de cabeza y las dos ríen.

Finalmente, nos ha parecido relevante analizar cómo y cuándo se usan las estrategias compensatorias del cambio de código y de la acuñación de palabras, extranjerización y traducción literal. Por lo que se refiere al cambio de código, este se produce en gran medida, en el caso de las interacciones de los estudiantes de alemán, entre alemán e inglés y no siempre es motivado. Por lo que se refiere a la acuñación de palabras, extranjerización y traducción literal, esta responde más bien a querer suplir lagunas léxicas:

Ejemplo 6. Jigsaw online díada B
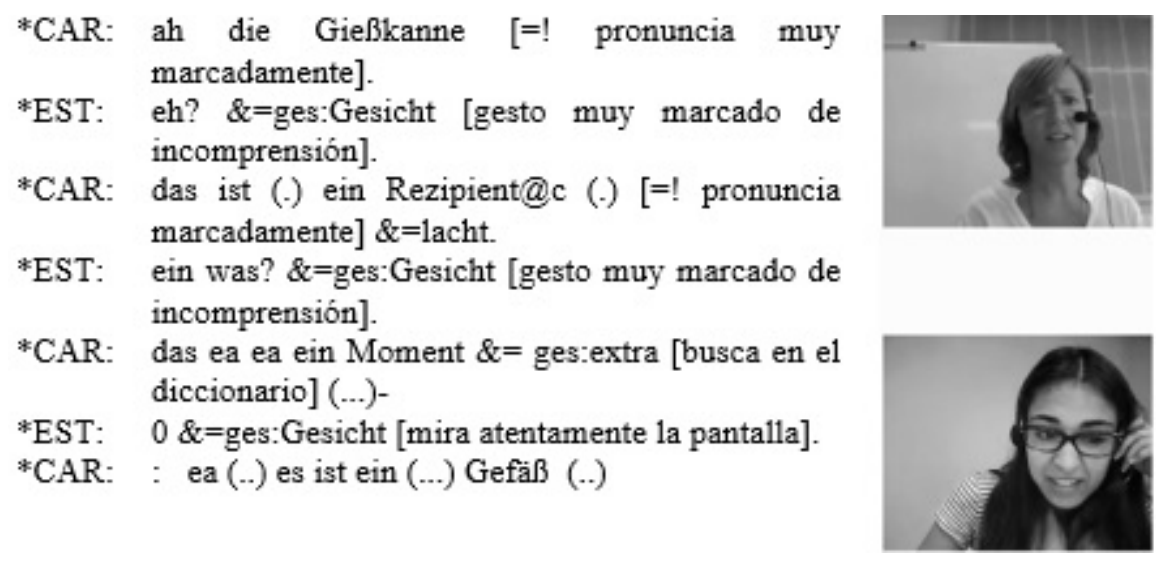

En el caso del ejemplo 6, la traducción literal ("Rezipient”) la utiliza CAR para explicar el objeto "Gießkanne" que EST no entiende. La risa tras la traducción literal muestra que es consciente de no usar la palabra adecuada y a continuación opta por buscar el término en el diccionario.

En el ejemplo 7, podemos ver como KAO ha acuñado la palabra "escombres" a partir de la palabra española "escombros" y la terminación francesa "-es", correspondiente al femenino plural. En la misma línea, se situaría el verbo "baler" que no existe en francés, puesto que la forma correcta es "balayer", y que surge a partir de la raíz del sustantivo "balai" y la terminación de los verbos de la primera conjugación francesa "-er". 
Ejemplo 7. Jigsaw online díada B
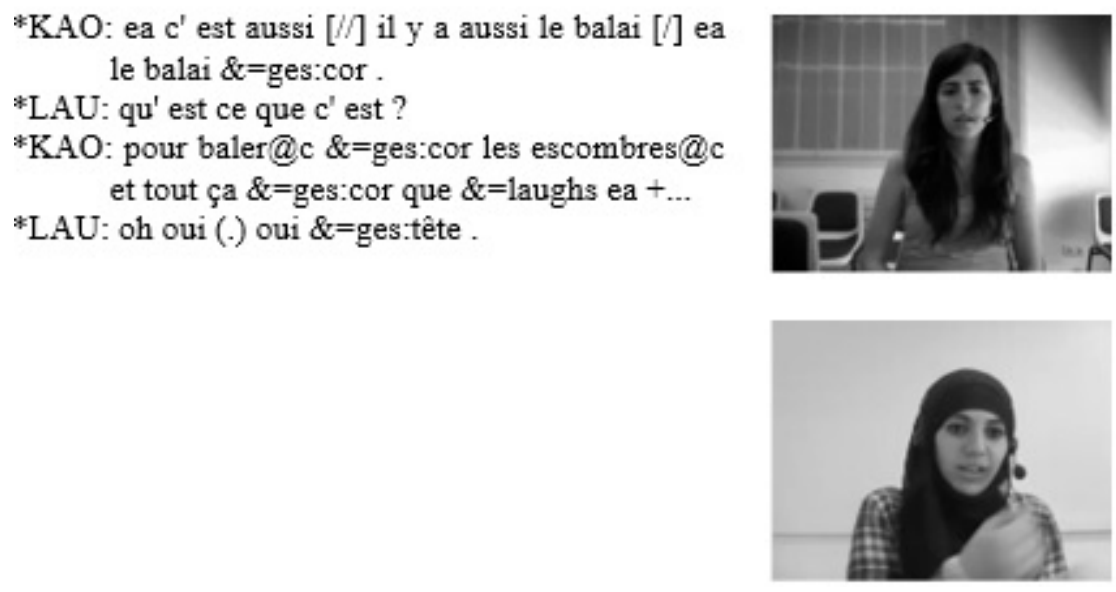

El cambio de código se da, en los diálogos en francés, para expresar la duda, el acuerdo o para introducir una petición de confirmación. Esta estrategia compensatoria no se usa generalmente en nuestro corpus como solución a la falta de vocabulario, ya que no sustituye a palabras o expresiones que los estudiantes desconocen, sino que se trata de elementos lingüísticos que estos conocen bien porque los usan en otros momentos de las interacciones. Así pues, al final de la secuencia del ejemplo 8, aparece la expresión coloquial "vale" que se utiliza para expresar acuerdo o aprobación en catalán y en español, y que se corresponde con las expresiones "d'accord” en francés estándar o “O.K." en francés coloquial.

Ejemplo 8. Jigsaw presencial díada B

\footnotetext{
*KAO: oui \&=ges:tête (.) [/] oui (.) ea c' est vrai parce que dans le sac à dos \&=ges:cor i1 $[/ / / /]$ nous peut mettre \&=ges:cor beaucoup plus de choses $\&=$ ges:cor .

${ }^{*}$ LAU: 0 \& $=$ ges:tête [asiente] .

*KAO: ea oui \& $\&$ ges tête $($.$) je crois$ que tu as raison.

*LAU: \&=laughs vale@eat .
}

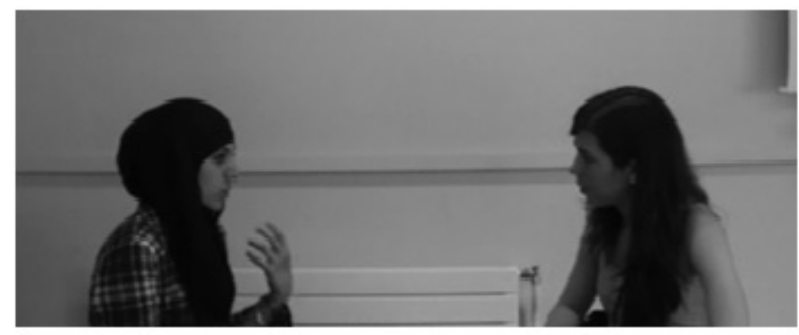




\section{DISCUSIÓN Y CONCLUSIONES}

A continuación, se discuten los resultados y el análisis presentado con el fin de responder a las preguntas de investigación que nos habíamos planteado.

Por lo que se refiere a la primera pregunta, es decir, a las estrategias comunicativas que se activan durante las tareas de interacción oral en la modalidad presencial y en la modalidad en línea, se observa que en los dos idiomas se usan más estrategias compensatorias que interaccionales en la modalidad cara a cara que en la modalidad a distancia.

Según nuestros datos, una parte muy importante de las estrategias compensatorias usadas en la modalidad cara a cara pertenece a la estrategia de mímica, que en nuestro estudio se corresponde con la gestualidad icónica, y que en las tareas realizadas en línea se usa en menor medida. Esta diferencia se podría explicar por la polifocalización de la atención a la que están sujetos los aprendientes en el momento de realizar una tarea por videoconferencia durante la cual deben estar atentos a su propia imagen, a la imagen de su interlocutor, a los documentos de trabajo y a las posibles búsquedas de léxico en línea. Esta polifocalización ha sido determinada también por otros autores como Holt, Tellier y Guichon (2015), Kerbrat-Orecchioni (2011), o Knight, Dooly y Barberà (2018). En las entrevistas retrospectivas que se realizaron, dos de las participantes de alemán manifestaron que en los diálogos realizados en línea se veían limitadas en cuanto al uso de mímica como estrategia comunicativa. Sin embargo, otras dos apuntaron a que el hecho de trabajar con varias pantallas abiertas de forma sincrónica no influyó sobre su capacidad para llevar a cabo la tarea.

Esta sería la diferencia más notable que se aprecia entre las dos modalidades que son objeto de nuestro estudio puesto que los otros aspectos importantes a destacar dependen más del tipo de tarea que de la modalidad en la que se ha llevado a cabo. Se confirman así en nuestro trabajo los resultados de Yanguas (2010) quien no pudo detectar diferencias entre la interacción cara a cara y la desarrollada a través de videoconferencia en su análisis. En nuestro propio estudio piloto (Delgar, 2015; Keim y Tortadès, 2015) tampoco se pudieron detectar diferencias significativas entre ambas modalidades.

Con respecto a la segunda pregunta de investigación, en nuestros datos el tipo de tarea que implica un mayor uso de estrategias es la tarea de tipo jigsaw. Teniendo en cuenta que este tipo de tareas es, como se ha mencionado anteriormente, más complejo que el ranking según los elementos establecidos por Baralt, Gilabert y Robinson (2014) para definir la complejidad de una tarea, no resulta sorprendente. Dado que el léxico relacionado con la información a compartir para poder realizar la segunda parte de la tarea no era conocido, las estrategias interaccionales que se activan con más frecuencia, tanto en alemán como en francés, están motivadas por la clarificación. Este resultado está en línea con el obtenido por Rosas (2018) que apunta a que las tareas de tipo jigsaw llevan a tener que resolver las lagunas léxicas 
de forma rápida y eficiente. En nuestros datos los aprendientes recurren para ello, en la modalidad cara a cara sobre todo, a la estrategia compensatoria de la mímica, que se usa de forma complementaria posiblemente para reforzar la explicación que se da verbalmente. En las entrevistas retrospectivas dos de las alumnas de francés y dos de las de alemán indican que se sirvieron de la estrategia de mímica para ayudarse a explicar algunos elementos léxicos. El uso combinado de gestualidad con otro tipo de estrategias comunicativas lo constatan también Isaeva y Fernández (2016) si bien en su caso se detecta sobre todo la combinación de búsqueda de palabra y gestualidad enfática y deíctica.

Si bien en las tareas de tipo ranking el uso de estrategias es menor, parece ser que las tareas utilizadas para nuestro análisis favorecen la construcción colaborativa del discurso en el caso del alemán, que es ligeramente superior en este tipo de tarea, y en francés el uso de pausas largas. Así mismo, tanto en francés como en alemán, se recurre más a la reestructuración que en el caso de las tareas de tipojigsaw. A partir de estas observaciones, podemos afirmar que, en función de la tarea, los estudiantes activan más algunos tipos de estrategias que otros y que, tal y como afirman Guichon y Nicolaev (2009, p. 1), "ciertas características de la tarea pueden tener un impacto en la producción oral de los aprendientes”.

Con relación a la tercera pregunta de investigación, hay algunas diferencias entre el uso de estrategias por parte de las estudiantes de alemán y los de francés. Así, la petición de ayuda es casi inexistente en las interacciones de los estudiantes de francés y es más notable en las llevadas a cabo en alemán. Esta diferencia podría estar relacionada con factores individuales dado que entre las estudiantes de alemán que participaron en el proyecto había un alto grado de complicidad y confianza que les permitía solicitar ayuda de forma explícita o implícita al buscar la forma de expresarse o al buscar una determinada palabra en la L3. En cambio, la estrategia de búsqueda se encuentra más representada en los diálogos en francés que en los de alemán. También en este caso el resultado podría explicarse con una variable individual puesto que la frecuencia de uso de esta estrategia es particularmente alta en el caso de uno de los estudiantes. Otra explicación podría ser que se debe a un mayor dominio de léxico en la L3 en el caso del francés. De hecho, en las entrevistas retrospectivas las estudiantes de alemán insisten en que el mayor problema al interaccionar de forma oral en alemán es activar de forma espontánea el léxico necesario.

Un resultado destacable en nuestro estudio es que las estrategias comunicativas no se activan de manera aislada, sino que ciertas estrategias se usan de manera articulada tejiendo secuencias interactivas complejas en muchos casos. Por ejemplo, la petición de clarificación en las tareas de tipo jigsaw da paso a la clarificación mediante la paráfrasis y/o mediante la repetición. $\mathrm{Y}$, a su vez, puede conllevar el uso de la estrategia compensatoria de aproximación y, muy frecuentemente, el uso de la mímica. Por otro lado, la estrategia de la pausa larga aparece asociada con la construcción colaborativa del discurso y/o la estrategia de reestructuración que se 
puede activar para la autocorrección o, simplemente, en paralelo con el razonamiento para encontrar la fórmula más adecuada. El análisis muestra además que, tanto en alemán como en francés, los aprendientes recurren a la acuñación de palabras, la extranjerización y la traducción literal para compensar lagunas léxicas mientras que el cambio de código suele usarse para expresar la duda, el acuerdo, la petición de confirmación, para marcar la autocorrección, y en momentos de más implicación emocional. En Dewaele y Salomidou (2017) se hace referencia a este componente emocional de los cambios de código, especialmente en hablantes multilingües. Cabe destacar que la estrategia de cambio de código se manifiesta mayoritariamente en el caso de las interacciones en alemán entre esta lengua y el inglés mientras que en las realizadas en francés se da siempre con la L1, es decir, con el español o el catalán. Este fenómeno podría explicarse por la proximidad existente entre una misma familia de lenguas.

Es necesario señalar que las estrategias relacionadas con evasión como son fingir comprensión o abandono del mensaje están poco representadas en nuestros datos. Sin embargo, en las entrevistas retrospectivas algunos de los participantes hacen mención de la necesidad de omitir en alguna ocasión la clarificación a fin de contribuir a la fluidez de la comunicación y la consecución de la tarea. Requeriría de posteriores análisis profundizar en estos momentos concretos de la interacción durante el desarrollo de la tarea.

Finalmente, algunas limitaciones de nuestro estudio son, por un lado, el posible sesgo de las investigadoras al transcribir la gestualidad icónica en las actividades realizadas en línea, si bien el encuadre generalmente permitía ver toda la parte superior del torso, así como los movimientos de brazos y manos. Por otro lado, los resultados son válidos para la población estudiada y no necesariamente transferibles a otras situaciones de aprendizaje, aunque, de hecho, nuestro estudio corrobora gran parte de las observaciones realizadas en estudios de estrategias durante la interacción oral presencial y en línea en lenguas adicionales. De cara a futuras investigaciones nos parece necesario destacar que el análisis detallado de la interacción muestra cómo los aprendientes de lenguas son agentivos (Knight, Barberà y Appel, 2017; Martín Peris, 2014) recurriendo a un entramado de recursos interaccionales que les permite alcanzar sus objetivos comunicativos de forma cooperativa con los interlocutores (González-Lloret, 2015), tanto de forma presencial como en línea.

\section{AGRADECIMIENTOS}

Las autoras agradecen el apoyo de la Dra. Sarah Khan y la Dra. Àngels Pinyana, investigadoras del Grupo de investigación 'Aprendizaje y Comunicación' de la Universidad de Vic-Universidad Central de Cataluña. 


\section{NOTAS}

1. Se solicitó el consentimiento por escrito de todos los estudiantes participantes para utilizar los datos de las grabaciones, así como las imágenes en publicaciones científicas relacionadas con el estudio realizado.

2. Estas tareas, son una adaptación de las tareas de Yanguas (2010, pp. 72-93), de Jeong (2011, pp. 51-69) y de Cotton et al. (2010, pp. 74 y 96).

\section{REFERENCIAS}

Baralt, M., Gilabert, R., y Robinson, P. (Eds.). (2014). Task Sequencing and Instructed Second Language Learning. London: Bloomsbury Academic.

Bygate, M. (2015). Domains and Directions in the Development of TBLT. Amsterdam: John Benjamins.

Chamot, A. U. (2004). Issues in Language Learning Strategy Research and Teaching. Electronic Journal of Foreign Language teaching, 1(1), 14-26. Recuperado de http://e-flt.nus.edu.sg/v1n12004/ chamot.pdf

Cosnier, J., y Develotte, C. (2011). Le face à face en ligne, approche éthologique. En C. Develotte, R. Kern y M.-N. Lamy, (Eds.), Décrire la conversation en ligne. Le face à face distanciel (27-50). Lyon: ENS Éditions.

Cotton, D. et al. (2010). Market Leader: intermediate business English course book. 3rd Edition. Harlow: Pearson Education.

Delgar, G. (2015). L'interaction orale en présentiel et à distance: une étude de cas en classe de français. Synergies Espagne, 8, 111-122. Recuperado de https://gerflint. fr/Base/Espagne8/delgar.pdf

Develotte, C., Kern, R., y Lamy, M.-N. (Eds.). (2011). Décrire la conversation en ligne. Le face à face distanciel. Lyon: ENS Éditions.

Dewaele, J. M., y Salomidou, L. (2017). Loving a partner in a Foreign Language. Journal of Pragmatics, 108, 116-130. doi: 10.1016/j.pragma.2016.12.009.
Dörnyei, Z., y Kormos, J. (1998). Problem-solving mechanisms in L2 Communication. A psycholinguistic perspective. Studies in Second Language Acquisition, 2O, 349-385.

González-Lloret, M. (2015). Conversation analysis in Computer-assisted Language Learning. CALICO Journal, 32(3), 569594. doi: 10.1558/cj.v32i3.27568.

Guichon, N., y Cohen, C. (2016). Multimodality and CALL. En F. Farr y L. Murray (Eds.), The Routledge Handbook of Language Learning and Technology (509-521). London: Routledge.

Guichon, N., y Nicolaev, V. (2009). Caractériser des tâches d'apprentissage et évaluer leur impact sur la production orale en L2. En C. Develotte, F. Mangenot. y E. Nissen, (Coords.), Actes du colloque Epal 2009 (Échanger pour apprendre en ligne: conception, instrumentation, interactions, multimodalité). Recuperado de https://bit.ly/2SxBLIO

Hampel, R., y Stickler, U. (2012). The use of videoconferencing to support multimodal interaction in an online language classroom. ReCALL, 24(2), 116-137. doi: http://dx.doi.org/10.1017/ So95834401200002X

Holt, B., Tellier, M., y Guichon, N. (2015). The use of teaching gestures in an online multimodal environment: the case of incomprehension sequences. Gesture and Speech in Interaction 4th Edition. Nantes. Recuperado de https://hal.archivesouvertes.fr/hal-01215770v2/document 
Isaeva, E., y Fernández-Villanueva, M. (2016). Gestures and Lexical Access Problems in German as Second Language. En M. Fernández-Villanueva y K. Jungbluth, (Eds.), Beyond Language Boundaries (93-113). Berlin: de Gruyter.

Jeong, N.-S. (2011). The Effects of Task Type and Group Structure on Meaning Negotiation in Synchronous ComputerMediated Communication. En L. Plonsky y M. Schierloh, (Eds.), Selected Proceedings of the 2009 Second Language Research Forum (51-69). Somerville, MA: Cascadilla Proceedings Project. Recuperado de http://www. lingref.com/cpp/slrf/2009/paper2524. pdf

Keim, L., y Tortadès, À. (2015). Comparación de la interacción oral de estudiantes de alemán L3 presenciales y online en una tarea de aula. RIED. Revista Iberoamericana de Educación a Distancia, 18(2), 325-353. doi: https://doi. org/10.5944/ried.18.2.13486

Kerbrat-Orecchioni, C. (2011). Conversations en présentiel et conversations en ligne: bilan comparatif. En C. Develotte, R. Kern y M.-N. Lamy, (Eds.), Décrire la conversation en ligne. Le face à face distanciel (173-195). Lyon: ENS Éditions.

Khan, S. (2010). Strategies and spoken production on three oral communication tasks. A study of high and low proficiency EFL learners. Universitat Autònoma de Barcelona. Recuperado de http://hdl. handle.net/10803/32083

Knight, J., Barberà, E. y Appel, C. (2017). A framework for learner agency in online spoken interaction tasks. ReCALL, 29(3), 276-293. doi: 10.1017/ So95834401700009X.
Knight, J., Dooly, M., y Barberà, E. (2018). Multimodal meaning making: navigational acts in an online speaking task. System, 78, 65-78. doi: 10.1016/j. system.2018.07.007.

Martín Peris, E. (2014). Un modelo de enfoque plurilingüe para la enseñanza de lenguas en la escuela. Linguarum Arena, 5, 47-66. Recuperado de https:// repositori.upf.edu/handle/10230/24775

Mondada, L. (2016). Challenges of Multimodality: Language and the Body in Social Interaction. Journal of Sociolinguistics, 2O(3), 336-366. doi: https://doi.org/10.1111/josl.1 12177

Pica, T., Kanagy, R., y Falodun, J. (1993). Choosing and Using Communication Tasks for Second Language Instruction and Research. En G. Crookeset y S. Gass, (Eds.), Tasks and Language Learning. Integrating Theory and Practice (9-34). Clevedon: Multilingual Matters.

Rosas, M. (2018). L2 learners' use of communication strategies as affected by the task type. Revista Signos: Estudios de lengua y literatura, 51(96), 107-131. doi: 10.4067/So718-09342018000100107.

Wigham, C. R. (2017). A multimodal analysis of lexical explanation sequences in web conferencing-supported language teaching. En B. O'Rourke y U. Stickler, (Eds.),Specialissue of Language Learning in Higher Education: Synchronous communication technologies in language and intercultural learning and teaching in higher education, 7(1), (81-108).

Yanguas, I. (2010). Oral computer mediated interaction between L2 learners:it's about time! Language Learning \& Technology, 14(3), 72-93. doi: 10125/44227. 


\section{PERFIL ACADÉMICO Y PROFESIONAL DE LAS AUTORAS}

Lucrecia Keim Cubas. Doctora en Filología Alemana por la Universidad de Bielefeld y profesora de la Facultad de Educación, Traducción y Ciencias Humanas de la Universidad de Vic-Universidad Central de Cataluña. Imparte docencia en el Grado de Traducción, Interpretación y Lenguas Aplicadas. Está vinculada a los programas de doctorado Traducción, Géneroy Estudios Culturales, así como al programa Innovación e Intervención Educativas. Coordina la línea de enseñanza y aprendizaje de lenguas extranjeras del grupo de investigación Aprendizaje y Comunicación (GRAC) <http:// urecerca.uvic.cat $/$ CawDOS?id=cafad3bbbb3b9879\&idioma=ca\&tipo=activ $>$. E-mail: lucrecia.keim@uvic.cat

Gemma Delgar Farrés. Doctora en Filología Francesa por la Universidad Autónoma de Barcelona y titular de la Licence de lettres modernes por la Universidad Paul-Valéry Montpellier 3. Es profesora de la Facultad de Educación, Traducción y Ciencias Humanas de la Universidad de Vic-Universidad Central de Cataluña donde imparte docencia en el Grado de Traducción, Interpretación y Lenguas Aplicadas. Es miembro del Grupo de Investigación en Aprendizaje y Comunicación (GRAC) y sus principales líneas de trabajo son la enseñanza y aprendizaje de lenguas extranjeras, y la lingüística aplicada $<\underline{h t t p: / / u r e c e r c a . u v i c . ~}$ cat/CawDOS?id=0941b27248120f58\&idioma $=$ ca\&tipo=activ $>$.

E-mail: gemma.delgar@uvic.cat

Dirección:

Facultad de Educación, Traducción y Ciencias Humanas

Universidad de Vic-Universidad Central de Cataluña

C. de la Laura, $\mathrm{n}^{0} 13$

08500 Vic (España)

Fecha de recepción del artículo: 09/10/2018

Fecha de aceptación del artículo: 05/12/2018

Fecha de aprobación para maquetación: 24/02/2019 


\section{ANEXO}

\section{Estrategias consideradas en este estudio}

\section{Estrategias interaccionales}

1. Hablar despacio

2. Verificación de comprensión

3. Clarificación mediante paráfrasis

4. Clarificación mediante repetición

5. Petición de repetir lentamente

6. Petición de clarificación

7. Petición de repetición

8. Fingir comprensión

9. Expresión de suposición

10. Expresión de incomprensión

11. Resumir lo que se ha entendido

12. Interrumpir para ofrecer una alternativa

13. Construcción colaborativa del discurso

14. Petición de espera

\section{Estrategias compensatorias}

1. Asunción de riesgos

2. (a) Acuñación de palabras, extranjerización y traducción literal / (b) Cambio de código

3. Petición de ayuda

4. Abandono del mensaje

5. Pausa larga

6. Reestructuración

7. Mascullar

8. Omisión

9. Búsqueda

10. Aproximación

11. Mímica

12. Evitación de riesgos

13. Simplificación discursiva 